\title{
PRIMARY (LOCALISED NON-FAMILIAL) CONJUNCTIVAL AMYLOIDOSIS: THREE CASE REPORTS
}

\author{
C. M. MOORMAN and B. McDONALD \\ Oxford
}

\begin{abstract}
SUMMARY
Conjunctival amyloidosis is an uncommon condition which usually arises secondary to chronic infection or trauma to the eye. It is rare to find significant deposits of amyloid involving the conjunctiva alone in the absence of a known antecedent infective, traumatic or hereditofamilial disorder. We report three patients with primary localised conjunctival amyloidosis. Each patient presented with a painless swelling in the lower fornix; in two this gradually extended to involve the bulbar and palpebral conjunctiva of both eyes with friable yellow deposits. Detailed clinical photographs illustrate the specific features of the disease. None of the patients had previous ocular pathology and bacterial, viral and fungal cultures of the conjunctiva were negative. There were no signs of inflammation. Conjunctival biopsies in each case showed large amorphous nodular deposits of amyloid within the substantia propria. Extensive systemic investigations including serum and urinary protein immunoelectrophoresis were normal. Follow-up ranged from 2 to 15 years after presentation. Treatment was symptomatic. As the typical features of amyloidosis present late in the course of conjunctival disease a diagnostic biopsy is often delayed. Once there is histopathological verification plasma cell disease must be excluded, although if the amyloid is limited to the conjunctiva systemic involvement is unlikely.
\end{abstract}

Primary localised amyloidosis involving the conjunctiva is rare. The first reported cases appeared in the Scandinavian literature as early as 1871 and in 1902 Herbert ${ }^{1}$ described a case of 'colloid degeneration' of the conjunctiva which appears to have been an example of conjunctival amyloidosis.

Correspondence to: Dr Brendan McDonald, MRC Schizophrenia Research Group, Department of Neuropathology, Radcliffe Infirmary NHS Trust, Woodstock Road, Oxford OX2 6HE, UK.
Localised conjunctival amyloidosis may arise spontaneously as a painless swelling in the fornix, or secondary to chronic infection, for example in trachoma. The primary tumour enlarges slowly and spreads to involve the bulbar and palpebral conjunctiva. The condition is most often unilateral but in long-standing cases can involve both eyes; it is not usually associated with systemic disease but multiple myeloma should be excluded. ${ }^{2-5}$

We report the clinical and histopathological features seen in three patients with symptomatic, primary localised non-familial amyloidosis confined to the conjunctiva.

\section{Case 1}

\section{CASE REPORTS}

A 55-year-old man presented to the Emergency Department with a 3 week history of an irritable, sticky right eye. Examination showed infiltration of the inferior fornix with subconjunctival yellow deposits and a small friable discharging lesion at the lateral canthus (Fig. 1a). There were no other conjunctival lesions and ocular examination was otherwise normal. The left eye was unaffected.

$\mathrm{He}$ was unable to recall any recent history of trauma; he did remember getting lime in both eyes 15 years previously but there were no complications from this episode. No other relevant ocular or past medical history was recorded.

A biopsy of the mass showed lobular sheets of acellular amyloid. The conjunctiva healed well and the patient is now symptom free and off all treatment.

\section{Case 2}

A 79-year-old woman was first seen in 1975 complaining of intermittent redness and soreness of both eyes. Examination showed conjunctival injection of both eyes with papillary hypertrophy of the 


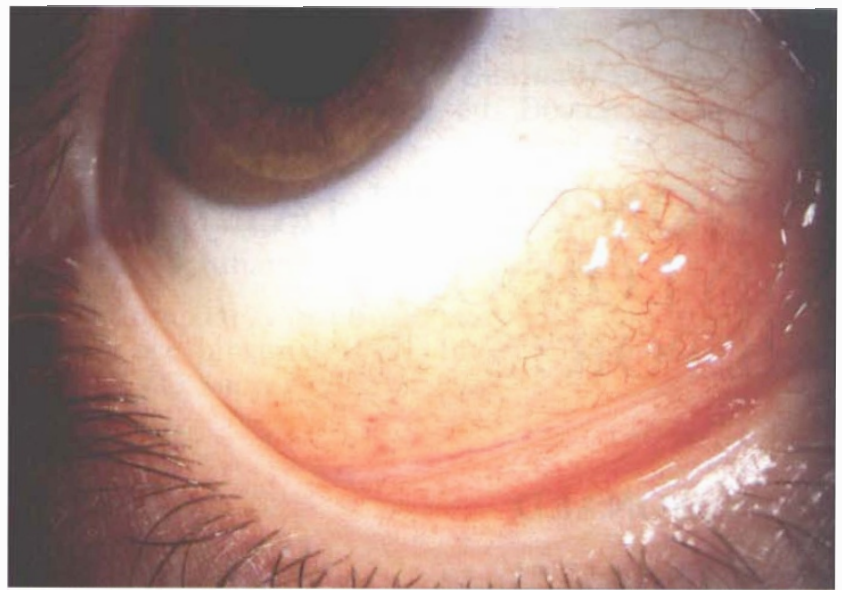

(a)

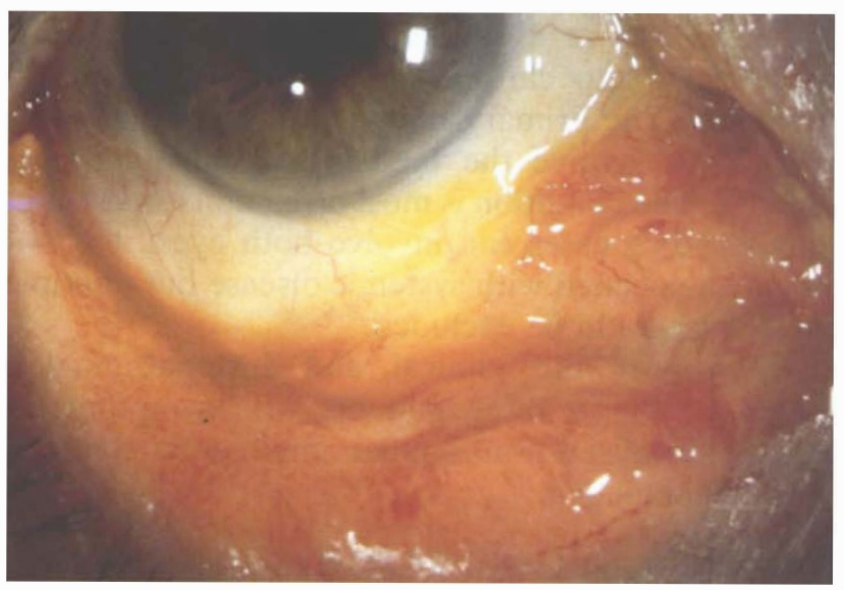

(c)

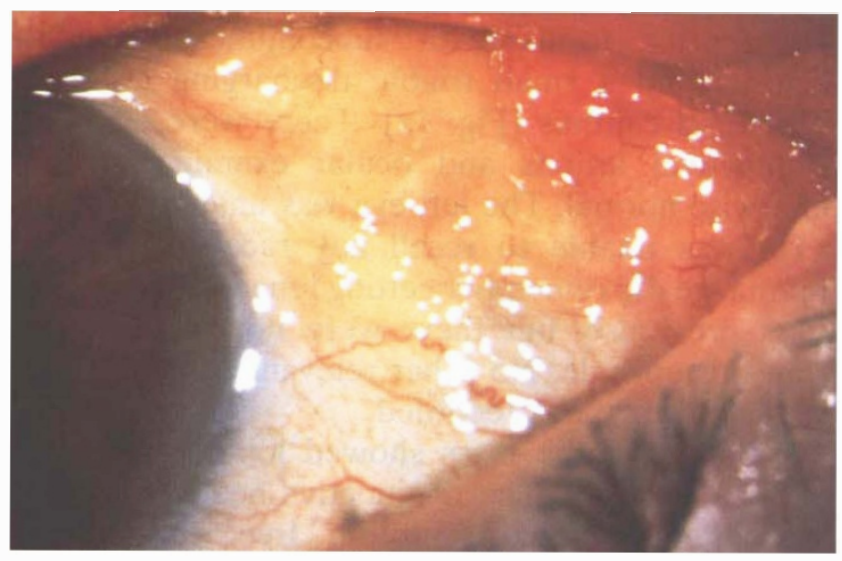

(e)

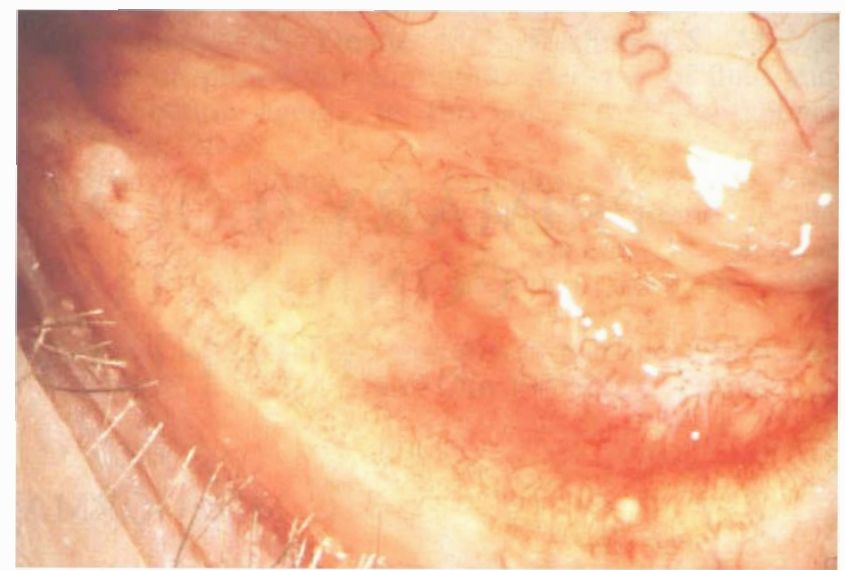

(b)

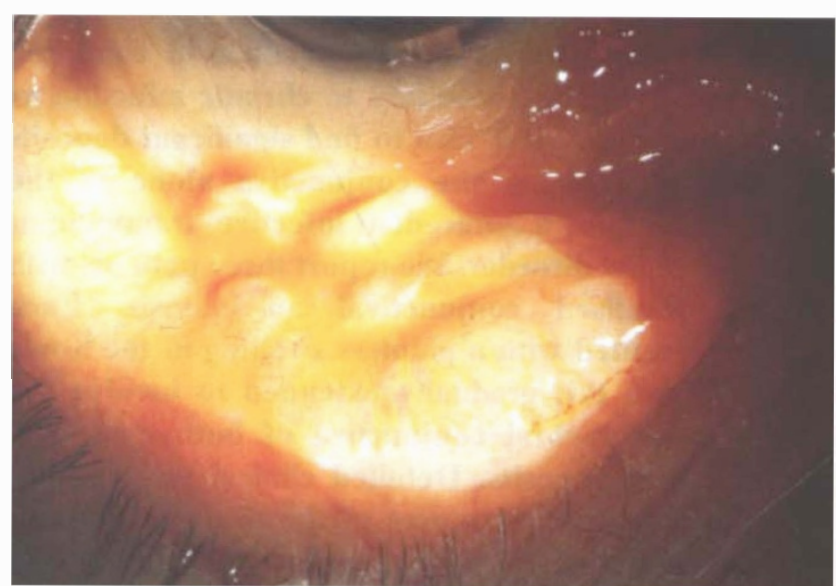

(d)

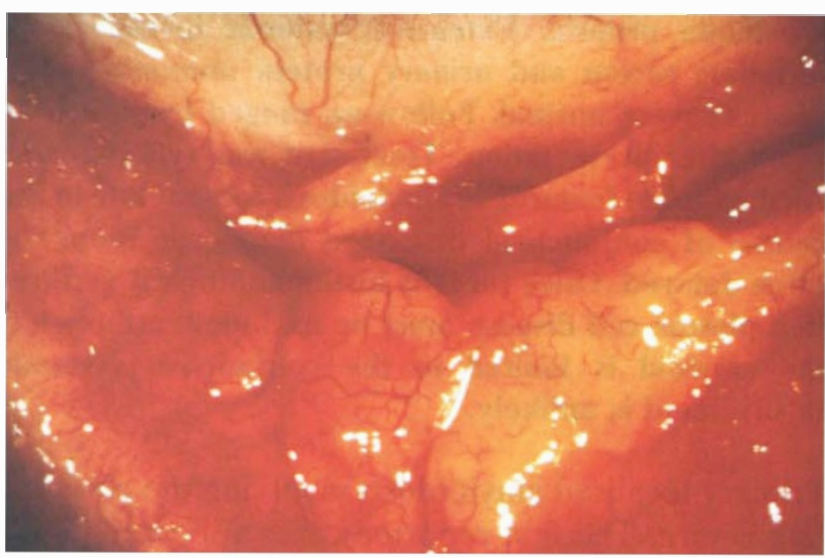

(f)

Fig. 1. Clinical features of conjunctival amyloidosis. (a) Case 1. Early infiltration of the bulbar conjunctiva and inferior fornix with amyloid. (Photograph taken after the biopsy site had healed.) (b) Case 2. Amyloid deposits in the inferior fornix and involving the punctum. There are multiple subconjunctival haemorrhages. (c) Case 2. Advanced conjunctival amyloid nodule involving the whole of the inferior fornix and extending to the caruncle. Note the friable-looking vessels and subconjunctival haemorrhages. (d) Case 3. Characteristic yellow, waxy amyloid deposits in the conjunctiva of the inferior fornix. (e) Case 3. Established subconjunctival nodular amyloidosis. (f) Case 3. Large yellow deposits of subconjunctival amyloid. 


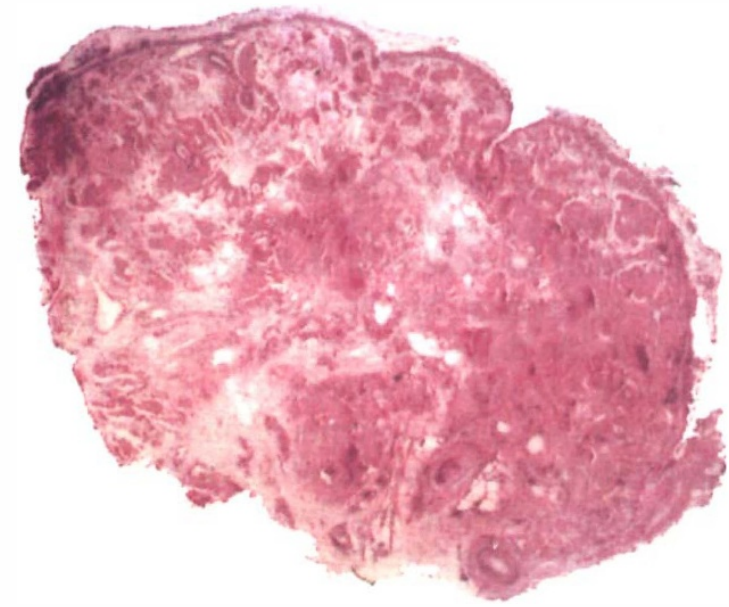

(a)

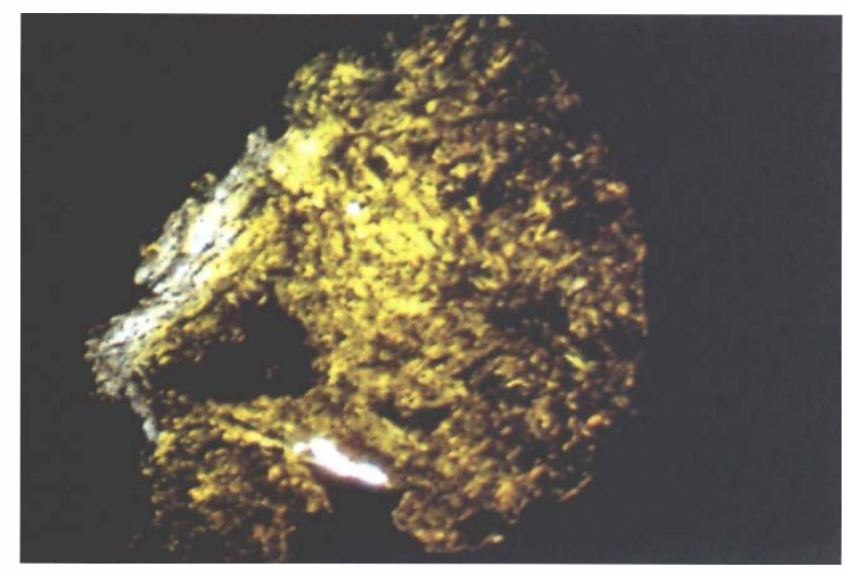

(c)

tarsal conjunctiva of all four lids. Ocular examination was otherwise normal.

A conjunctival biopsy at the time showed nonspecific papillary hypertrophy. Her general health was good and past ocular and medical history were unremarkable. Systemic haematology, biochemistry and serology studies (including protein electrophoresis and Rheumatoid Factor) and local virology, bacteriology and fungal cultures were negative. The patient's symptoms were relieved by a course of guttae prednisolone $0.5 \%$ b.d. and guttae sodium cromoglycate p.r.n. to both eyes.

Over the next 10 years the clinical signs became more florid and were complicated by intermittent spontaneous subconjunctival haemorrhages (Fig. 1b). A trial of topical antibiotics (tetracycline and neomycin) did not alleviate the soreness or alter the clinical appearance. By 1992 large yellow deposits were visible beneath the conjunctiva of both lower fornices and the right caruncle was heavily infiltrated (Fig. 1c). The eyelids and cornea are not involved.

Biopsy of the right inferior forniceal conjunctiva showed deposition of granular amyloid deposits in

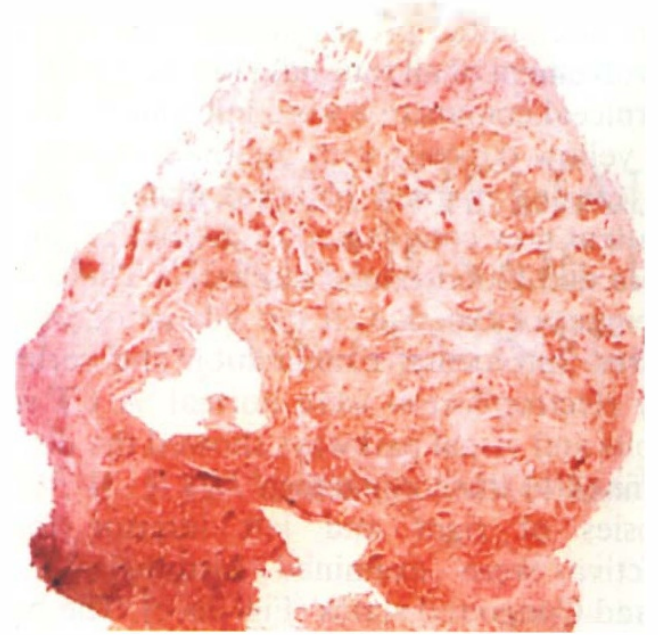

(b)

Fig. 2. (a) Low magnification $(\times 75)$ photomicrograph of the second biopsy of case 3 stained using haematoxylin and eosin stain. (b) Congo red stained section of the same biopsy, and (c) after being viewed with polarised light, showing the characteristic apple-green birefringence of amyloid.

the substantia propria. Immunohistochemically the amyloid showed staining for amyloid-P protein.

The patient remains in good health and is currently asymptomatic; she is no longer using topical treatment for her disease.

\section{Case 3}

A 72-year-old man presented in May 1982 with a 6 month history of a painless mass in the right lower lid which was gradually increasing in size. Examination revealed a nodular growth involving the medial tarsal conjunctiva of both upper and lower lids and yellow, waxy deposits in the lower forniceal conjunctiva (Fig. 1d). Ocular examination was otherwise normal with no evidence of infection or inflammation. The left eye was normal.

Systemic investigations (including FBC, ESR, LFTs, serum and urinary protein immunoelectrophoresis and chest radiograph) were normal and cultures for herpes simplex virus and acid-fast bacilli were negative. A conjunctival biopsy showed nodular amyloidosis. Over the next 2 years the lesions continued to increase in size and involved the left eye; when the patient was reviewed in February 1991 
(9 years after initial presentation) there was extensive involvement of all four lids (Fig. 1e, f). The tarsal and forniceal conjunctiva were infiltrated with large, friable yellow deposits with multiple small haemorrhages causing marked bilateral medial ectropion. The patient now complained of discomfort, bilateral epiphora and bloodstained discharge. There was no involvement of the skin of the lids or other orbital structures. Intraocular examination and serum and urinary electrophoresis were normal. A CT scan of the orbits and visual pathways showed the disease to be confined to the conjunctiva.

Biopsies of right and left inferior forniceal conjunctiva were examined histologically using $\mathrm{H} \& \mathrm{E}$ and Congo red stains (Fig. 2a-c). The biopsies showed large amorphous nodular deposits of amyloid within the substantia propria which demonstrated apple-green birefringence to polarised light following Congo red staining of the tissue. A light infiltrate of lymphocytes, plasma cells and eosinophils was present in the stroma surrounding the amyloid deposits. Immunohistochemical staining showed positive staining of the amyloid by antisera binding to amyloid-P protein. Antibodies binding to amyloid-A protein, immunoglobulin heavy/light chain and gelsolin failed to show a positive staining reaction, even after formic acid pre-treatment of the tissue sections. Review of the previous biopsy and immunohistochemical staining of the tissue confirmed the diagnosis of nodular 'tumour forming' amyloidosis of the conjunctiva. The patient is being treated symptomatically with guttae hypromellose as required.

\section{DISCUSSION}

Corneal amyloidosis in the form of lattice corneal dystrophy, familial subepithelial amyloidosis and polymorphic amyloid degeneration has been well described in the literature; the deposits contain amyloid-P component which is a polypeptide found in all forms of amyloid. Unlike conjunctival amyloid, there does not appear to be any convincing relationship between the known amyloid proteins and localised corneal amyloid. Although our specimens failed to show a reaction with antiserum for amyloid protein AL, recent studies have shown that localised conjunctival amyloid can be classified as AL type. ${ }^{6}$

Primary conjunctival amyloidosis is difficult to diagnose clinically in the early stages; the yellow, waxy deposits which bleed easily are diagnostic but these are not obvious initially. Until there are deposits visible in the conjunctiva, a biopsy may not seem indicated. Once there is histopathological verification, neoplastic plasma cell disease should be excluded, although if the amyloid is limited to the conjunctiva it is most probably a strictly localised process without systemic involvement. Amyloid-A protein, which is present in the secondary amyloidoses and in familial Mediterranean fever, was not identified in the biopsy specimens ${ }^{7}$ and none of these patients had a positive family history. This, combined with the fact that these patients remain clinically well and have normal systemic investigations, reduces the likelihood of systemic involvement. The issue of whether to investigate further by performing a rectal biopsy to exclude systemic amyloid deposits was discussed with each patient but they declined further investigation.

Two patients required long-term topical steroids and lubricants for relief of their symptoms. Various surgical procedures have been described in the past, for example en bloc or piecemeal removal of the involved tissues, but the extent of infiltration with friable deposits of amyloid with a tendency to bleed make this suitable for limited cases only. Patrinely et al. ${ }^{8}$ described a debulking procedure in advanced cases whereby the subconjunctival deposits of amyloid were scraped down to bare tarsus or sclera and a soft bandage contact lens was used to prevent apposition of raw surfaces and symblepharon formation. Recurrence of amyloid deposits did occur after 6-12 months. ${ }^{8}$

\section{REFERENCES}

1. Herbert H. Colloid degeneration of the conjunctiva. Trans Ophthalmol Soc UK 1902;22:261-6.

2. Behal ML. Secondary amyloid infiltration around the limbus. Br J Ophthalmol 1964;48:622-3.

3. Howard GM. Amyloid tumours of the orbit. $\mathrm{Br} \mathrm{J}$ Ophthalmol 1966;50:421-5.

4. Smith ME, Zimmerman LE. Amyloidosis of the eyelid and conjunctiva. Arch Ophthalmol 1966;75:42-50.

5. Knowles D, Jakobiec F, Rosen M, Howard G. Amyloidosis of the orbit and adnexae. Surv Ophthalmol 1975; 19:367-84.

6. Sandgren O. Ocular amyloidosis, with special reference to the hereditary forms with vitreous involvement. Surv Ophthalmol 1995;40:173-96.

7. Klintworth GK. Proteins in ocular disease. In: Garner A, Klintworth GK, editors. Pathobiology of ocular disease. New York: Marcel Dekker, 1994:973-1033.

8. Patrinely JR, Koch DD. Surgical management of advanced ocular adnexal amyloidosis. Arch Ophthalmol 1992;110:882-5. 